\title{
REVIEW
}

\section{Advantages of Ambulatory Blood Pressure Monitoring in Assessing the Efficacy of Antihypertensive Therapy}

\author{
Alejandro De la Sierra
}

To view enhanced content go to www.cardiologytherapy-open.com

Received: April 13, 2015 / Published online: June 16, 2015

(c) The Author(s) 2015. This article is published with open access at Springerlink.com

\section{ABSTRACT}

The cumulative evidence in the past three decades situates ambulatory blood pressure monitoring (ABPM) as a central element in diagnosing and predicting the prognosis of subjects with hypertension. However, for various reasons, this diagnostic and prognostic importance has not been translated in equal measure into making decisions or guiding antihypertensive treatment. Mean 24-h, daytime, and night-time blood pressure estimates, the occurrence of divergent phenotypes between clinic measurements, and ABPM, as well as the main elements that determine blood pressure variability over $24 \mathrm{~h}$, especially night-time dipping, are all elements that in addition to providing evidence for patient prognosis, can be used to guide antihypertensive treatment follow-up enabling greater precision in defining the effect of the

Electronic supplementary material The online version of this article (doi:10.1007/s40119-015-0043-1) contains supplementary material, which is available to authorized users.

\section{A. De la Sierra $(\bowtie)$}

Hospital Mútua Terrassa, Universidad de Barcelona, Terrassa, Barcelona, Spain

e-mail: adelasierra-@mutuaterrassa.cat drugs. In recent years, specific indices have been developed using 24-h monitoring, evaluate the duration of treatment action, the homogeneity of the effect over the monitoring period, and its possible effects on variability. In future controlled clinical trials on antihypertensive therapies it is necessary to evaluate the effects of those treatments on hard endpoints based on therapy guided by ABPM.

Keywords: Ambulatory blood pressure monitoring; Antihypertensive agents; Blood pressure variability; Circadian blood pressure profile; Hypertension; Morning blood pressure surge; Morning hypertension; Nocturnal hypertension

\section{INTRODUCTION}

Non-invasive clinic blood pressure (BP) measurement dates back to the early twentieth century and corresponds to the invention of the mercury sphygmomanometer by the Italian physicist Scipione Riva-Rocci, and the description of arterial sounds by the Russian physician Nikolai Korotkoff. Since then, 
changes in the procedure for clinic measurement have been few and have mostly been based on the appearance of automatic or semi-automatic devices that have used a plethysmographic method instead of the classical auscultatory method described by Korotkoff. Part of this change has been motivated by restrictions on using mercury in health care devices.

The two ways of measuring out-of-clinic BP, self-measurement at home and ambulatory BP monitoring (ABPM), were developed to obtain BP measurements outside health care settings, since those settings have a significant influence on BP in some individuals. Furthermore, especially in the case of ABPM, obtaining a higher number of measurements in a period containing the main sources of BP variability (activity/rest) provides a more precise approximation of an individual's true BP.

ABPM research has been particularly focused on epidemiological and diagnostic aspects. In this regard, the main indicators obtained during the course of ABPM correlate better with an individual's organ damage and cardiovascular prognosis [1-4]. Moreover, regarding diagnostic aspects, ABPM has enabled two new phenotypes to be defined, white-coat hypertension (HTN) and masked HTN, both of great clinical interest $[5,6]$.

Applying ABPM to therapeutic assessment has had less of an impact, both on the part of the investigators as well as on the part of the investment made by the sponsors of antihypertensive drugs and devices. A large part of this is motivated by the fact that the regulatory authorities continue to consider clinic BP measurement as the central element for approving antihypertensive drugs and devices. Nevertheless, the BP measurement indicators obtained using ABPM, the patient phenotypes during hypertensive treatment, dipping patterns, and BP variability over $24 \mathrm{~h}$ of monitoring, are of equally high interest when analyzing the effects of antihypertensive therapies [7]. Furthermore, using ABPM enables a single assessment of some aspects of that treatment, such as the duration of action by calculating specific indices that evaluate the duration and the homogeneity of the effect.

This article describes the main indicators, direct or derived, from monitoring itself or combined between ABPM and clinic measurement that may be of interest in patients during antihypertensive treatment. This article is based on previously conducted studies and does not involve any new studies of human or animal subjects performed by the author.

\section{MEAN 24-H, DAYTIME, AND NIGHT-TIME BP ESTIMATORS}

The mean 24-h, daytime, and night-time indicators have classically been the most used for both the relationship between ambulatory $\mathrm{BP}$ and cardiovascular prognosis, as well as for assessing the antihypertensive effect of drugs. Daytime BP, or BP during the period of activity, was one of the first parameters studied, since it is considered the closest to in-office BP. The current guidelines for diagnosing and treating HTN from the National Institute for Health and Care Excellence (NICE) of the British Government advises practicing ABPM during the day to confirm the HTN diagnosis when the clinic figures are high [8]. Daytime BP has also been the first used to assess the "white-coat" effect. In the first analyses of the Spanish ABPM Registry, up to $30 \%$ of patients without treatment $[9,10]$ and nearly $35 \%$ of those treated with clinic BP figures greater than or equal to $140 / 90 \mathrm{mmHg}$ [11] presented normal daytime BP figures (below 135/85 mmHg). 
One study has used daytime BP to guide antihypertensive treatment adjustment and monitoring in comparison with clinic BP [12]. Thus, patients with a clinic diastolic BP greater than or equal to $95 \mathrm{mmHg}$ were randomized to using daytime BP or clinic BP to guide treatment adjustments. Upon ending the study, the daytime BP-guided monitoring was associated with a lower use of antihypertensive drugs; with no differences observed in the organ damage measured using the degree of left ventricular hypertrophy.

The mean BP of all measurements made over $24 \mathrm{~h}$ of monitoring is considered to be the one that provides better information. A larger number of measurements are included in its calculation. As such, it is less affected by sporadic situations that may arise during the day or night or by sporadic errors. It takes into account daytime activity, work, and the changes in pressure caused by those activities, as well as rest and the quality of it, and the night-time pressure dip. Its relationship with cardiovascular prognosis, as well as the presence and severity of organ damage, are clearly better than clinic pressure. From a therapeutic point of view, several meta-analyses have evaluated the correlation between the decrease in clinic BP or 24-h BP induced by antihypertensive treatment. Thus, in one of them that included 44 studies with more than 5000 patients, the mean decreases in clinic BP were 19/10 $\mathrm{mmHg}$, while those corresponding to the 24-h figures were $13 / 8 \mathrm{mmHg}$. The percentage of $24-\mathrm{h}$ BP reductions compared to clinic BP was $65 \%$ and $81 \%$, respectively, for systolic and diastolic BP [13].

Another conclusion reached in that metaanalysis was that the definitions of responders and patients achieving BP control were not able to be extrapolated to the values obtained in 24-h ABPM. Thus, the final in-office BP values $(143 / 90 \mathrm{mmHg})$ were only slightly better than the normal values ( $<140 / 90 \mathrm{mmHg})$, suggesting that a high percentage of patients managed to achieve BP control. Conversely, the final 24-h $\mathrm{BP}$ values $(139 / 86 \mathrm{mmHg})$ were clearly above the limits of normal $(<130 / 80 \mathrm{mmHg})$, suggesting that achieving 24 -h control was far below the clinic control achievement. These results were later confirmed in another metaanalysis that included studies with clinic measurement, home measurement, and ABPM. The BP reduction was greater in the clinic than at home, and higher at home than in the 24-h values [14].

Night-time BP measurements have been progressively acquiring more importance. Of all the indicators obtained during $\triangle B P M$, it is the one that is best correlated with the prognosis [1-4]. Its main advantage is that it can be considered the baseline BP (the one that is specified for tissue perfusion in a state of rest). In addition, the fact that it is generally measured at rest gives it higher reproducibility and less variability, which makes it easier to correlate it with organ damage and prognosis. The data from the Spanish ABPM Registry [4], as well as the various prospective cohort databases [15], indicate that of all the BP indicators (clinic, daytime, night-time, and 24-h) it is the one that is best independently correlated with the prognosis (Fig. 1). The main disadvantages are that it requires an exact definition of the rest period, it may be affected by the presence of a daytime rest (nap) [16], and it is equally affected by the quality of sleep [17], especially in patients who repeatedly wake up during the night or who have sleep apnea. 


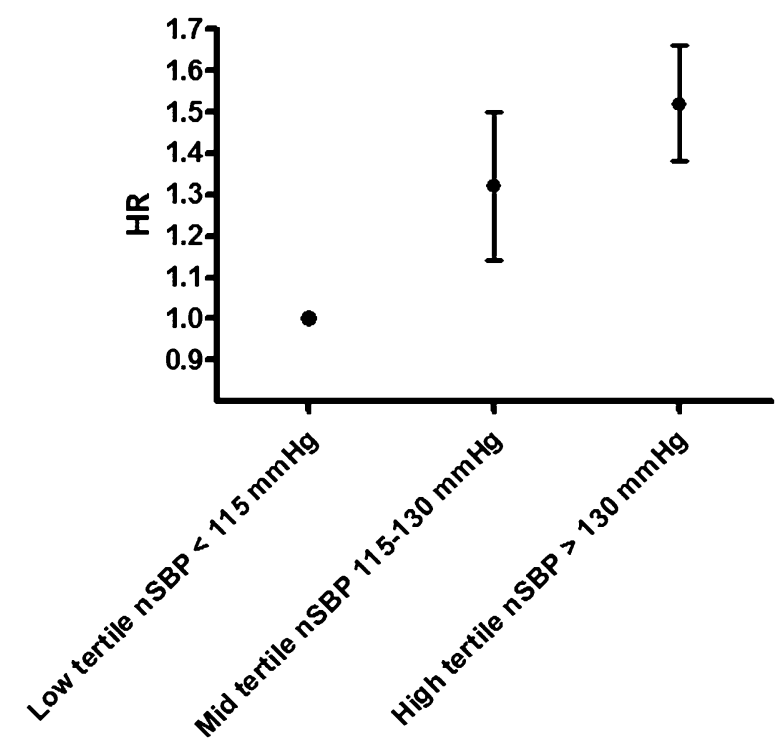

Fig. 1 Risk of cardiovascular events (non-fatal myocardial infarctions, non-fatal strokes, or cardiovascular deaths) in high-risk patients based on the tertile distribution of nSBP. The risk increases $32 \%$ and $50 \%$, respectively, for the middle and high tertiles. Data extracted from [4]. nSBP night-time systolic blood pressure, HR Hazard ratio

\section{PATIENT PHENOTYPES}

Jointly using clinic measurement and ABPM has resulted in the creation of new patient categories that have expanded the hypertensive/normotensive dichotomy. Thus, the consistency between normal figures in clinic BP and those obtained through ABPM is called normotension, whereas the consistency between high figures in the clinic and ABPM constitute sustained HTN. The two new types represent the presence of discrepancies between both forms of measurement. Thus, high figures in the office and normal ones in ABPM constitute the phenotype known as white-coat HTN or isolated clinic HTN. This category, in principle, restricted to the diagnosis of patients without treatment, is also used in patients on treatment in whom ABPM figures are controlled, but not those in the clinic. At the opposite extreme are individuals with normal clinic BP figures but with high ABPM figures. This situation is known as masked HTN in untreated individuals or as masked uncontrolled hypertension (MUCH) in those who are on treatment.

Though there is a general consensus that the presence of masked HTN or MUCH confers a risk that may be comparable to that of sustained HTN [18, 19] (possibly from a population perspective the risk is even greater, given that the individuals who do not know they belong to this group are mostly not being treated and outside of health care oversight), there are still serious doubts about whether white-coat HTN carries an increased risk and whether it requires treatment [20, 21]. In general, longitudinal studies have observed that individuals with white-coat HTN present a risk of cardiovascular events similar to normotensive individuals, although in some cases, an increase in cerebrovascular accidents has been detected. Similarly, its association with organ damage has been described as similar to that of normotensive individuals in some studies, or with a higher prevalence of cardiac or renal damage in others. In many cases, it is hard to reach a conclusion since, even with the whitecoat HTN diagnosis, these individuals have higher ABPM figures than normotensive individuals and, in addition, a very high percentage go on to develop sustained HTN in its progression [22].

The prevalence of these divergent phenotypes depends in large part on the parameter used to define them and the study population. In patients with antihypertensive treatment and high clinic BP figures, white-coat HTN varies between $27 \%$, if normality is required in all periods (daytime, night-time, and 24-h), and $45 \%$ if only daytime normality is considered [11] (Fig. 2). For its part, the prevalence of $\mathrm{MUCH}$ in patients with normal clinic BP figures also varies 
between $24 \%$, if only daytime figures are considered, and $49 \%$ if it is defined based on elevation in any of the indicators (daytime, night-time, or 24-h; Fig. 3) [23].

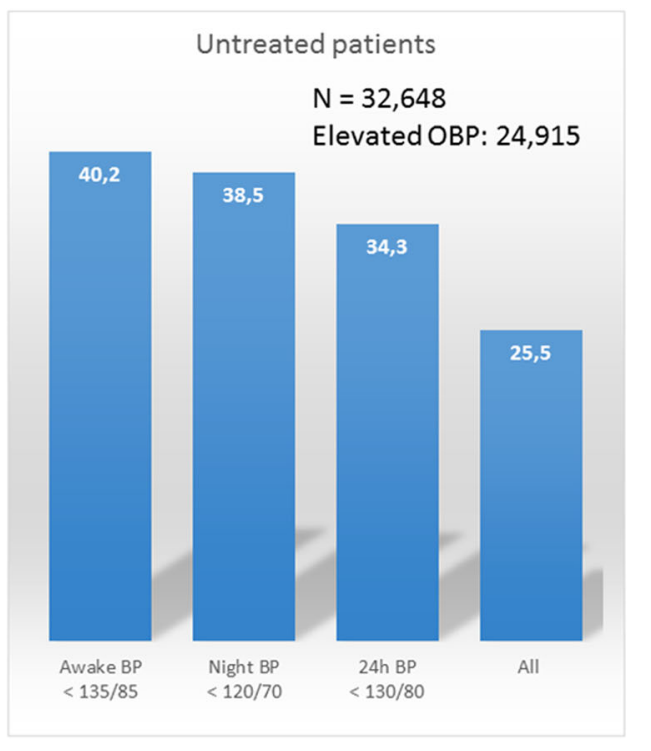

Fig. 2 Prevalence of white-coat hypertension in patients in the Spanish Ambulatory BP Monitoring Registry with or without treatment and with clinic BP values greater than or equal to $140 / 90 \mathrm{mmHg}$. The prevalence depends on which

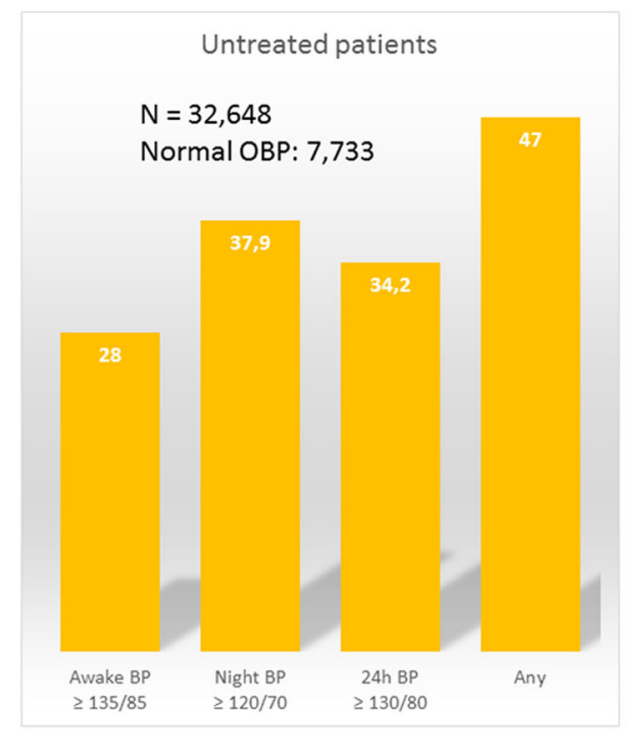

Fig. 3 Prevalence of masked hypertension in patients in the Spanish Ambulatory BP Monitoring Registry with (right) or without (left) antihypertensive treatment and normal clinic BP $(<140 / 90 \mathrm{mmHg})$. The prevalence
The clinical features that are associated with these divergent phenotypes are, in the case of white-coat HTN, old age, the female sex, the absence of other risk factors such as smoking or

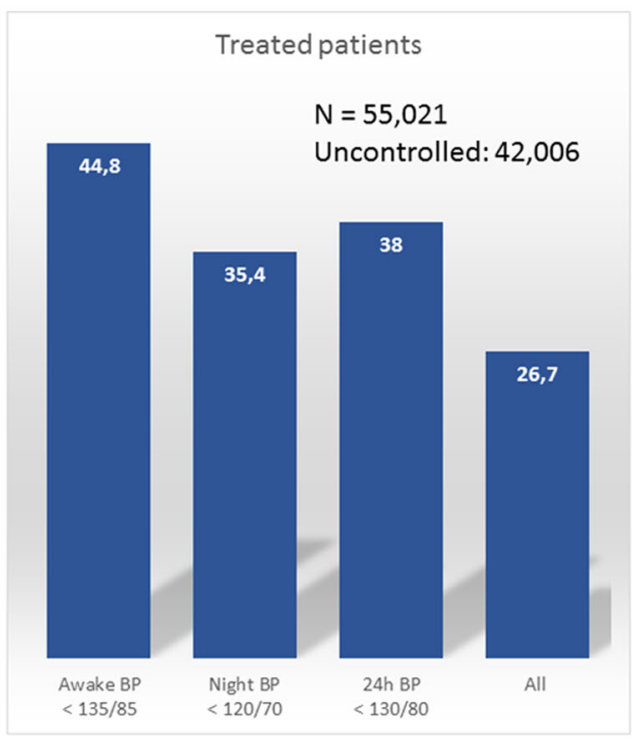

parameter is used (daytime, night-time, or 24-h BP, or the normalcy all of them). $B P$ Blood pressure, $O B P$ Office blood pressure

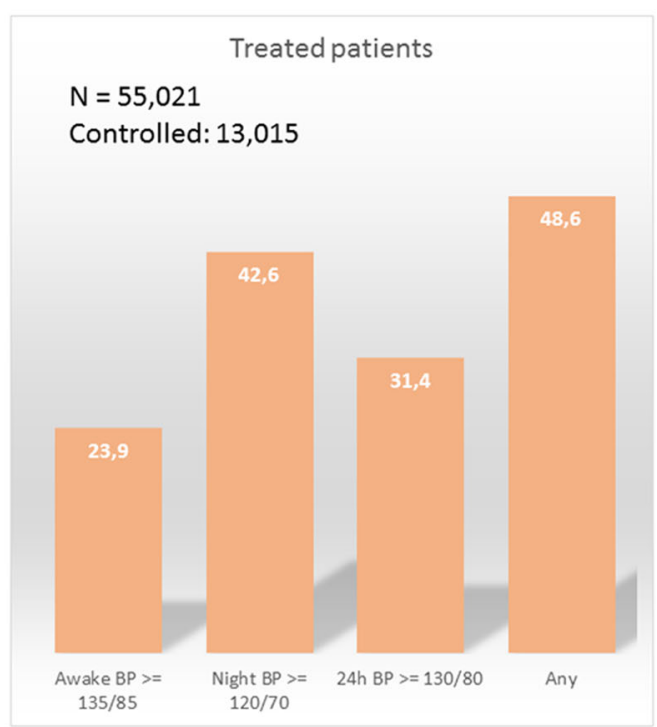

depends on which parameter is used (daytime, night-time, or 24-h BP, or any of them). BP Blood pressure, $O B P$ Office blood pressure 
diabetes, and cardiac or renal organ damage [9, 24]; whereas youth, the male sex, smoking, diabetes, and the presence of organ damage are correlated with a higher probability of presenting masked HTN [23, 25]. Obesity deserves particular mention, as it seems to be correlated, albeit weakly, with both white-coat HTN and masked HTN $[9,11,23]$. The interpretation is that obesity may be an element that decreases the accuracy of clinic measurement and, as such, has an impact not just on phenotype, but also on discrepancies between measurements. However, it should be recognized that these clinical associations, although significant, have little predictive ability in individuals and in no case should substitute ABPM in diagnosing a specific patient. Other features or the physician's intuition should also not have any impact. Thus, the sensitivity and specificity of the clinical suspicion related by the physician for diagnosing white-coat HTN presented very low values in relation to the definitive diagnosis made by using ABPM [9].

At this time, there is no absolute consensus on the need for antihypertensive treatment in patients with divergent phenotypes. All the observational studies demonstrate that masked HTN or MUCH result in a high risk of developing future cardiovascular events. As such, it seems logical to think that those patients are suitable for starting antihypertensive therapy. Nevertheless, no study has demonstrated that this treatment will improve the prognosis in those patients; therefore, the therapeutic decision is completely empirical. Even more uncertainties exist about the follow-up for these patients, including target figures or regularity of repeating ABPM [26].

As for subjects with white-coat HTN, as previously mentioned, there are discrepancies between the likelihood of having a major cardiovascular event or not. Moreover, treatment in those patients, even reducing the BP figures, seems to have little impact on the BP figures obtained by ABPM. The current guidelines recommend antihypertensive treatment in high-risk patients with known cardiovascular disease or with hypertensive organ damage. In the rest, a close follow-up and early detection of the appearance of sustained HTN seems to be the best option [27].

\section{DIPPING PATTERNS}

The decrease in BP caused by rest and sleep, usually at night, has a favorable impact on reducing the pressure burden related to the organ damage. Almost 40 years ago, it was described that some patients in whom this night-time dip was less pronounced (the threshold has been established at $10 \%$ versus the daytime values) had a worse risk profile and a higher probability of developing cardiovascular events and death [28]. In general, four dipping patterns have been described based on this night-time decrease. The most common pattern in the healthy population is known as the "dipper" pattern and it represents between a $10 \%$ and $20 \%$ decrease from daytime values. The extreme "dipper" pattern exceeds this 20\% and, even though it has been described as associated with a risk of cerebrovascular accident in the Asian population, a clearly deleterious effect has not been demonstrated on the prognosis in Westerners. Conversely, a decrease below 10\%, known as a "non-dipper" pattern (recently the term "reduced dipper" has been proposed), or an increase in BP during rest, known as a "riser" pattern, have both been associated with a worse prognosis and related with organ damage [28, 29]. 
The data from the Spanish ABPM Registry have allowed us to determine that the prevalence of these "deleterious" patterns is very high, approaching $50 \%$ of untreated patients and exceeding this figure in those on treatment (Fig. 4). Old age, the female sex, obesity, diabetes, and a history of previous cardiovascular disease are associated with an inadequate decrease in both treated and untreated patients. In treated patients, increasing the number of drugs also results in a higher probability of presenting a non-dipper or riser pattern [30, 31].

The main problem in assessing the dipping pattern is its association with night-time BP levels. Both elevated night-time BP as well as inadequate nocturnal dip have been related with a worse prognosis. Nevertheless, the fact remains that both situations are intimately related, which makes it difficult to separate their independent effects. A separate assessment of both phenomena has enabled these uncertainties to be cleared up. Thus, there have been patients with inadequate night-time BP dip who despite everything present normal

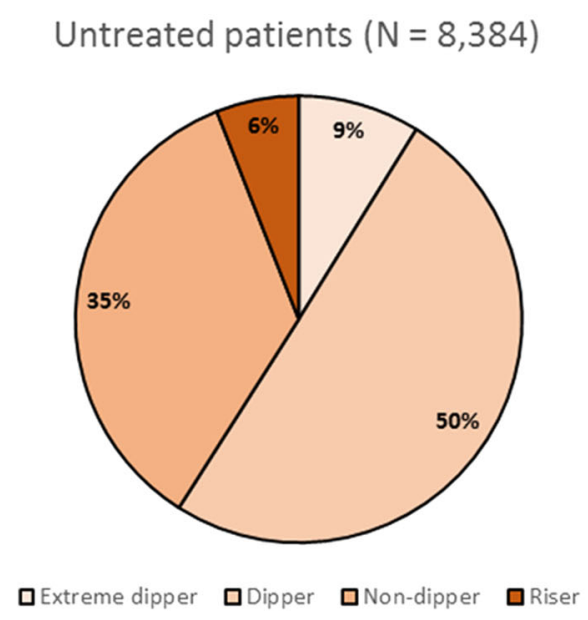

Fig. 4 Distribution of dipping patterns in patients without hypertensive treatment (left) or with hypertensive treatment (right) included in the Spanish Ambulatory Blood Pressure Monitoring Registry. The presence of an night-time BP figures, and by contrast, there have been patients with night-time HTN but with a night-time BP dip above $10 \%$. In this analysis, the non-dipper pattern in the absence of night-time HTN was associated with the female sex, impaired kidney function, and a history of cardiovascular events, whereas nighttime HTN in the presence of a normal pattern was associated with the male sex, diabetes, and asymptomatic organ damage (microalbuminuria and left ventricular hypertrophy). Obviously the worse risk profile was observed in patients who presented both night-time HTN and a non-dipper pattern [32].

\section{MORNING HTN AND MORNING SURGE}

After the sleep-induced night-time BP dip, the morning surge that accompanies waking is a physiological phenomenon. However, some studies have observed that an exaggerated morning BP surge is associated with a higher rate of cardiovascular events [33]. The hormone changes that affect cortisol and catecholamines,

\section{Treated patients $(\mathrm{N}=34,563)$}

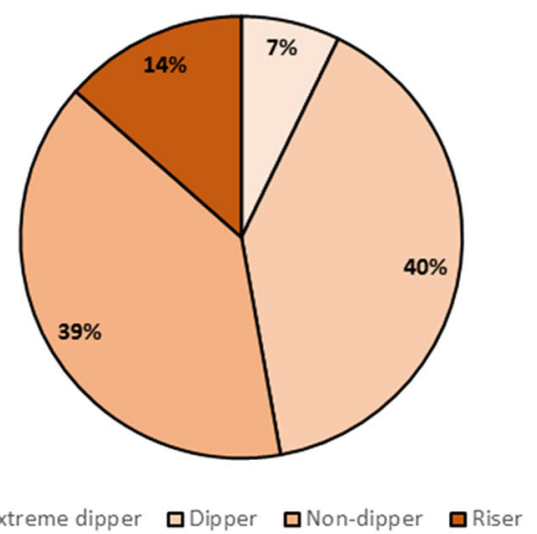

inadequate night-time dip (riser or non-dipper pattern) is around $50 \%$ in patients without treatment and exceeds this figure in those treated 
the increase in heart rate, and the higher platelet aggregability that happen in these morning hours are a rationale to explain this phenomenon [34].

Another parameter of interest intimately related to the morning surge is known as morning HTN, which consists of high BP figures obtained just after waking. A recent study in patients being treated for HTN demonstrated that the BP figures obtained in the first hour of the morning through selfmeasurement had a greater prognostic impact than clinic BP figures. Values above $145 \mathrm{mmHg}$ were associated with a higher rate of cardiovascular events [35].

The main problems that arise when evaluating the phenomena of the morning surge and morning HTN are, on one hand, that most studies have been carried out in a Japanese population. Data in a Mediterranean population suggest that the morning surge in this population is less pronounced than in the Japanese population (De la Sierra; personal communication); thus its prognostic impact is presumably less. On the other hand, morning surge and morning HTN are influenced not only by physiological or pathophysiological circumstances, but also by type of treatment, its posology, and the duration of action of the drugs. Thus, if we take into account that most drugs are administered in the morning, this morning surge coincides with the end of the period of the therapeutic window, and therefore only those drugs with a longer half-life will significantly reduce these parameters.

Although there are not many comparative studies, not all antihypertensive drugs ensure $24 \mathrm{~h}$ of coverage. Olmesartan and telmisartan among the angiotensin receptor blockers, lisinopril among the angiotensin-converting enzyme inhibitors, amlodipine among the calcium blockers, and chlorthalidone among the diuretics are probably the drugs with the longest duration of antihypertensive effect in their respective classes. Some others with shorter coverage times may see that time extended with pharmaceutical modifications that slow down their absorption.

\section{BP VARIABILITY}

$\mathrm{BP}$ is a dynamic parameter that fluctuates based on several circumstances, some intrinsic and other extrinsic [36]. Long-term BP variability may be determined through successive visits. This variability has a prognostic impact, especially in predicting cerebrovascular accidents [37]. ABPM enables short-term fluctuations to be assessed. Some of these, caused by the activity/rest rhythm, have already been mentioned and are part of the night-time dip patterns. However, pressure fluctuations within one of these periods (daytime and especially at night) also have a prognostic impact. They can be evaluated by calculating the standard deviation in one of the periods separately and by using indices that take into account these deviations, and project the calculation over the entire 24 -h period [36].

In recent years, there has been a growing interest in assessing the impact of antihypertensive treatment on this short-term variability determined by ABPM. Initial studies have used modifications in the posology of the drugs (administering part or all of the treatment at night) and assessed their impact on the dipping pattern. Thus, it has been demonstrated that this night-time administration promotes a larger night-time dip and, as such, a proportion of non-dipper or riser patients becomes dippers. In one of these studies, this phenomenon was associated with a better cardiovascular prognosis [38]. 


\section{THERAPEUTIC INDICES OBTAINED USING ABPM}

In addition to all the above-described parameters used to assess the effect of antihypertensive therapy, some mathematical indices have been developed that combined potency, duration of action, and homogeneity of effect. This allows for a better assessment of the effects of antihypertensive treatment.

The first of these indices is the trough-topeak $(T / P)$ ratio. Interest in this ratio appeared nearly two decades ago and consists of calculating the ratio between the decrease in BP obtained in the hours just before the end of the therapeutic window (with drugs administered once a day between 22 and $24 \mathrm{~h}$ after administration) and the maximum effect calculated after several hours (between 4 and $6 \mathrm{~h}$ after administration). In theory, the closer it is to unity, the greater the homogeneity of effect, suggesting that the residual effect of the drug is close to its maximum effect. However, the $T / P$ ratio has two significant problems. The first is a result of poor reproducibility, caused by the need to extract short periods of monitoring from a 24-h ABPM that may be influenced by external factors. Thus, the peak period or maximum effect may coincide with a postprandial rest (nap), which will magnify it, or with a period of higher physical or mental activity, which will minimize it. For its part, calculating the trough effect may coincide with the last hours of sleep or with waking, both circumstances that may change it. In addition, the $T / P$ ratio does not take into account the magnitude of the antihypertensive effect, so that minimal decreases in BP in the peak will be associated with high $T / P$ indices (placebos usually have a $T / P$ index around 1) [39].

The second index that measures homogeneity of effect is called the smoothness index (SI) [40]. It is calculated based on the hourly reductions in $\mathrm{BP}$, corrected by the standard deviation of those reductions. Thus, the greater the magnitude of the hourly reduction and the smaller the differences between those reductions (less variability), the higher the resulting number will be. The higher the SI value is, the higher the drug potency and the greater the homogeneity of effect. Some studies have described an ability of the SI to predict changes in organ-damage parameters (left ventricular mass and carotid intima-media thickness) caused by the treatment [41].

One last index proposed very recently is the treatment-on-variability index (TOVI), calculated using the ratio between the 24-h decrease in BP and the change in the weighted standard deviation (hourly standard deviation calculated separately during the daytime and night-time periods and later weighted based on the duration of each of those periods) [42]. A recent study using clinical trial databases with several monotherapies and one combination therapy demonstrated a greater effect of the combination therapy and the amlodipine monotherapy based on that index, in comparison with two angiotensin receptor blockers and one angiotensin-converting enzyme inhibitor [42].

\section{RESISTANT HTN}

Between $10 \%$ and $15 \%$ of patients with HTN do not manage to normalize their $\mathrm{BP}$ values despite treatment with 3 or more antihypertensive drugs. They fall under the category of resistant HTN [24, 43]. Most of them are referred to a specialist clinic, and despite extensive diagnostic work and a search for secondary causes that explain their high $\mathrm{BP}$, the reason why the BP values cannot be normalized has not been explained. These patients have more 
organ damage [44] and a worse prognosis [45] than other patients with HTN.

In a broad study stemming from patients in the Spanish ABPM Registry, one-third of 8295 patients who could be categorized as resistant hypertensive had normal 24-h BP figures, where resistance was due to a white-coat effect. Compared with truly resistant hypertensive patients, the organ damage in these patients was less and the cardiovascular prognosis better [24].

The need to demonstrate that the BP values obtained using ABPM are undoubtedly high in resistant hypertensive patients was recently supported by the appearance of more invasive therapies, such as renal sympathetic denervation or baroreflex stimulation as a treatment for patients with resistant HTN [46]. The first studies on renal denervation have demonstrated a significant decrease in BP that was not confirmed in the recent SIMPLICITY HTN-3 study (ClinicalTrials.gov number, NCT01418261), where patients with resistant HTN required ABPM confirmation [47].

\section{CONCLUSION}

ABPM should be considered the standard measurement of BP, a starting point for assessing and treating patients with $\mathrm{HTN}$, as some recent guidelines recommend $[5,6,8,48]$. A large body of evidence demonstrating a close epidemiological relationship between the indicators obtained using ABPM, organ damage, and cardiovascular prognosis has not been followed by an equally significant translation of the impact of ABPM as a guide to antihypertensive therapy. It has only been in recent years where, in part due to scientific interest and in part due to requirements from regulatory agencies, the need for assessing the effects of the primary treatments on 24-h BP has been emphasized. The consistency of the mean estimators (24-h, daytime, and night-time BP figures), the new phenotypes of white-coat HTN and masked HTN, the importance of the dipping status and BP variability, and the appearance of specific indices for treatment assessment have made it so that ABPM should today be considered an essential element for guiding antihypertensive treatment, thereby enabling a more personalized medicine adapted to the patient. The main barriers for a more widespread use of ABPM are related to several factors including costs and reimbursement, acceptability and complexity in the interpretation of some estimators. However, they can be easily solved. More validated devices are available in the market with reduced prices, and the acceptance of patients and health workers in considering results as a better guidance for diagnosis and treatment has considerably increased. Moreover, different software and website platforms have developed to provide rapid and easy reports containing the most important estimators of clinical validity. Some experiences in several countries, including Australia, Ireland, Italy, and Spain have demonstrated that ABPM could be implemented in almost all clinical settings, from primary care to reference units, and even in community pharmacies. This will improve HTN management in the very near future.

\section{ACKNOWLEDGMENTS}

This supplement has been sponsored by Menarini. The named author meets the International Committee of Medical Journal Editors (ICMJE) criteria for authorship for this manuscript, takes responsibility for the integrity 
of the work as a whole, and has given final approval for the version to be published.

Conflict of interest. Alejandro De la Sierra declares honoraria for participation in scientific meetings funded by Abbott, Daiichi-Sankyo, Lacer, Menarini, Merck, Sharp \& Dohme, and Pfizer.

Compliance with ethics guidelines. This article is based on previously conducted studies and does not involve any new studies of human or animal subjects performed by the author.

Open Access. This article is distributed under the terms of the Creative Commons Attribution Noncommercial License which permits any noncommercial use, distribution, and reproduction in any medium, provided the original author(s) and the source are credited.

\section{REFERENCES}

1. Kikuya M, Ohkubo T, Asayama K, et al. Ambulatory blood pressure and 10-year risk of cardiovascular and noncardiovascular mortality: the Ohasama Study. Hypertension. 2005;45:240-5.

2. Dolan E, Stanton A, Thijs L, et al. Superiority of ambulatory over clinic blood pressure measurement in predicting mortality: the Dublin Outcome Study. Hypertension. 2005;46:156-61.

3. Sega R, Facchetti R, Bombelli M, et al. Prognostic value of ambulatory and home blood pressures compared with office blood pressure in the general population. Follow-up results from the Pressioni Arteriose Monitorate e Loro Associazioni (PAMELA) study. Circulation. 2005;111:1777-83.

4. De la Sierra A, Banegas JR, Segura J, Gorostidi M, Ruilope LM. Ambulatory blood pressure monitoring and development of cardiovascular events in highrisk patients included in the Spanish ABPM Registry. J Hypertens. 2012;30:713-9.

5. O'Brien E, Parati G, Stergiou G, et al. European Society of Hypertension position paper on ambulatory blood pressure monitoring. J Hypertens. 2013;31:1731-68.

6. Parati G, Stergiou G, O'Brien E, et al. European Society of Hypertension practice guidelines for ambulatory blood pressure monitoring. J Hypertens. 2014;32:1359-66.

7. Schmieder RE, Ruilope LM, Ott C, Mahfoud F, Bohm M. Interpreting treatment-induced blood pressure reductions measured by ambulatory blood pressure monitoring. J Human Hypertens. 2013;27:715-20.

8. National Institute for Health and Clinical Excellence (NICE). Hypertension. The clinical management of primary hypertension in adults. Clinical Guideline 127. 2011. Available at: www.nice.org.uk/guidance/ CG127. Accessed 11 June 2015.

9. Vinyoles E, Felip A, Pujol E, et al. Clinical characteristics of isolated clinic hypertension. J Hypertens. 2008;26:438-45.

10. Vinyoles E, Rodríguez-Blanco T, de la Sierra A, et al. Isolated clinic hypertension: diagnostic criteria based on 24-h blood pressure definition. J Hypertens. 2010;28:2407-13.

11. Banegas JR, Segura J, Sobrino J, et al. Effectiveness of blood pressure control outside the medical setting. Hypertension. 2007;49:62-8.

12. Staessen JA, Byttebier G, Buntinx F, Celis H, O'Brien ET, Fagard R. Antihypertensive treatment based on conventional or ambulatory blood pressure measurement. A randomized controlled trial. JAMA. 1997;278:1065-72.

13. Mancia G, Parati G. Office compared with ambulatory blood pressure in assessing response to antihypertensive treatment: a meta-analysis. J Hypertens. 2004;22:435-45.

14. Ishikawa J, Carroll DJ, Kuruvilla S, Schwartz JE, Pickering TG. Changes in home versus clinic blood pressure with antihypertensive treatments: a metaanalysis. Hypertension. 2008;52:856-64.

15. Roush GC, Fagard RH, Salles GF, et al. Prognostic impact from clinic, daytime, and night-time systolic blood pressure in nine cohorts of 13,844 patients with Hypertension. J Hypertens. 2014;32:2332-40.

16. Bursztyn M, Mekler J, Wachtel N, Ben-Ishay D. Siesta and ambulatory blood pressure monitoring. Comparability of the afternoon nap and night sleep. Am J Hypertens. 1994;7:217-21.

17. Verdecchia P, Angeli F, Borgioni C, Gattobigio R, Reboldi G. Ambulatory blood pressure and 
cardiovascular outcome in relation to perceived sleep deprivation. Hypertension. 2007;49:777-83.

18. Bobrie G, Clerson P, Menard J, Postel-Vinay N, Chatellier G, Plouin PF. Masked hypertension: a systematic review. J Hypertens. 2008;26:1715-25.

19. Ohkubo T, Kikuya M, Metoki H, et al. Prognosis of "masked" hypertension and "white-coat" hypertension detected by 24-h ambulatory blood pressure monitoring 10-year follow-up from the Ohasama study. J Am Coll Cardiol. 2005;46:508-15.

20. Verdecchia P, Reboldi GP, Angeli F, et al. Short- and long-term incidence of stroke in white-coat hypertension. Hypertension. 2005;45:203-8.

21. De la Sierra A. Definition of white coat hypertension: ambulatory blood pressure, selfmeasured blood pressure or both? Hypertension. 2013;62:16-7.

22. Mancia G, Bombelli M, Facchetti R, et al. Long-term risk of sustained hypertension in white-coat or masked hypertension. Hypertension. 2009;54:226-32.

23. Banegas JR, Ruilope LM, de la Sierra A, et al. High prevalence of masked uncontrolled hypertension (MUCH) in people with treated hypertension. Eur Heart J. 2014;35:3304-12.

24. De la Sierra A, Segura J, Banegas JR, et al. Clinical features of 8,295 patients with resistant hypertension classified on the basis of ambulatory blood pressure monitoring. Hypertension. 2011;57:898-902.

25. Gorostidi M, Vinyoles E, Banegas JR, de la Sierra A. Prevalence of white-coat and masked hypertension in national and international registries. Hypertens Res. 2015;38:1-7.

26. Muxfeldt ES, Fiszman R, de Souza F, Viegas B, Oliveira FC, Salles GF. Appropriate time interval to repeat ambulatory blood pressure monitoring in patients with white-coat resistant hypertension. Hypertension. 2012;59:384-9.

27. Mancia G, Fagard R, Narkiewicz K, et al. 2013 ESH/ ESC Guidelines for the management of arterial hypertension. The Task Force for the management of arterial hypertension of the European Society of Hypertension (ESH) and of the European Society of Cardiology (ESC). J Hypertens. 2013;31:1281-357.

28. O'Brien E, Sheridan J, O'Malley K. Dippers and nondippers. [Letter]. Lancet. 1988;2:397.

29. Ohkubo T, Hozawa A, Yamaguchi J, et al. Prognostic significance of the nocturnal decline in blood pressure in individuals with and without high 24-h blood pressure: the Ohasama study. J Hypertens. 2002;20:2183-9.

30. De la Sierra A, Redón J, Banegas JR, et al. Prevalence and factors associated with circadian blood pressure patterns in hypertensive patients. Hypertension. 2009;53:466-72.

31. De la Sierra A, Segura J, Gorostidi M, Banegas JR, de la Cruz JJ, Ruilope LM. Diurnal blood pressure variation, risk categories, and antihypertensive treatment. Hypertens Res. 2010;33:767-71.

32. De la Sierra A, Gorostidi M, Banegas JR, Segura J, de la Cruz JJ, Ruilope LM. Nocturnal hypertension or non-dipping: which is better associated with the cardiovascular risk profile? Am J Hypertens. 2014;27:680-7.

33. Kario K, Pickering TG, Umeda Y, et al. Morning surge in blood pressure as a predictor of silent and clinical cerebrovascular disease in elderly hypertensives: a prospective study. Circulation. 2003;107:1401-6.

34. Kario K. Morning surge in blood pressure and cardiovascular risk: evidence and perspectives. Hypertension. 2010;56:765-73.

35. Kario K, Saito I, Kushiro T, et al. Home blood pressure and cardiovascular outcomes in patients during antihypertensive therapy: primary results of HONEST, a large-scale prospective, real-world observational study. Hypertension. 2014;64:989-96.

36. Parati G, Ochoa JE, Lombardi C, Bilo G. Assessment and management of blood-pressure variability. Nat Rev Cardiol. 2013;10:143-55.

37. Rothwell PM, Howard SC, Dolan E, et al. Prognostic significance of visit-to-visit variability, maximum systolic blood pressure, and episodic hypertension. Lancet. 2010;375:895-905.

38. Hermida RC, Ayala DE, Mojón A, Fernández JR. Decreasing sleep-time blood pressure determined by ambulatory monitoring reduces cardiovascular risk. J Am Coll Cardiol. 2011;58:1165-73.

39. Omboni S, Parati G, Zanchetti A, Mancia G. Calculation of trough-to-peak ratio of antihypertensive treatment from ambulatory blood pressure: methodological aspects. J Hypertens. 1995;13:1105-12.

40. Parati G, Omboni S, Rizzoni D, Agabiti-Rosei E, Mancia G. The smoothness index: a new reproducible and clinically relevant measure of the homogeneity of the blood pressure reduction by treatment for hypertension. J Hypertens. 1998;16:1685-91. 
41. Rizzoni D, Muiesan ML, Salvetti $M$, et al. The smoothness index, but not the trough-to-peak ratio predicts changes in carotid artery wall thickness during antihypertensive treatment. J Hypertens. 2001;19:703-11.

42. Parati G, Dolan E, Ley L, Schumacher H. Impact of antihypertensive combination and monotreatments on blood pressure variability: assessment by old and new indices. Data from a large ambulatory blood pressure monitoring database. J Hypertens. 2014;32:1326-33.

43. Calhoun DA, Jones D, Textor S, et al. Resistant hypertension: diagnosis, evaluation and treatment: a scientific statement from the American Heart Association Professional Education Committee of The Council for High Blood Pressure Research. Hypertension. 2008;51:1403-19.

44. De la Sierra A, Banegas JR, Oliveras A, et al. Clinical differences between resistant hypertensives and patients treated and controlled with three or les drugs. J Hypertens. 2012;30:1211-6.
45. Daugherty SL, Powers JD, Magid DJ, et al. Incidence and prognosis of resistant hypertension in hypertensive patients. Circulation. 2012;125:1635-42.

46. Oliveras A, de la Sierra A. New developments in the diagnosis and management of resistant hypertension. Curr Med Chem. 2012;19:1210-8.

47. Bhatt DL, Kandzari DE, O'Neill WW, et al. A controlled trial of renal denervation for resistant hypertension. N Engl J Med. 2014;370:1393-401.

48. Piper MA, Evans CV, Burda BU, Margolis KL, O'Connor E, Whitlock EP. Diagnostic and predictive accuracy of blood pressure screening methods with consideration of rescreening intervals: a systematic review for the US Preventive Services Task Force. Ann Intern Med. 2015;162:192-204. 